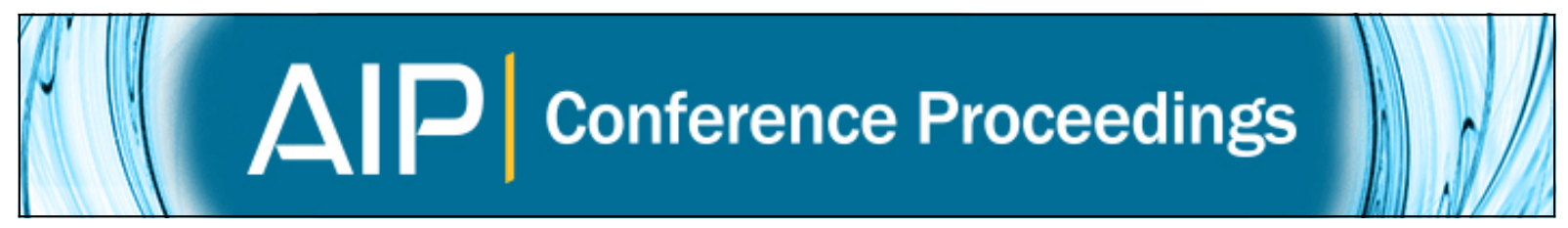

\title{
Spectral classification of worldwide locations using SMR indexes
}

Rubén Núñez, Chen Jin, Ignacio Antón, and Gabriel Sala

Citation: AIP Conference Proceedings 1766, 090007 (2016); doi: 10.1063/1.4962113

View online: http://dx.doi.org/10.1063/1.4962113

View Table of Contents: http://scitation.aip.org/content/aip/proceeding/aipcp/1766?ver=pdfcov

Published by the AIP Publishing

Articles you may be interested in

Classification of odontocete using spectral properties of echolocation clicks

J. Acoust. Soc. Am. 131, 3493 (2012); 10.1121/1.4709199

Coarse classification of acoustic signals using temporal and spectral characteristics

J. Acoust. Soc. Am. 121, 3046 (2007); 10.1121/1.4781747

Semi-automatic classification of bird vocalizations using spectral peak tracks

J. Acoust. Soc. Am. 120, 2974 (2006); 10.1121/1.2345831

Seabed classification from acoustic profiling data using the similarity index

J. Acoust. Soc. Am. 111, 794 (2002); 10.1121/1.1433812

Location and classification of plosive consonants using expert knowledge and neural net classifiers

J. Acoust. Soc. Am. 84, S60 (1988); 10.1121/1.2026396 


\title{
Spectral Classification of Worldwide Locations Using SMR Indexes
}

\author{
Rubén Núñez ${ }^{\text {a) }}$ Chen Jin, Ignacio Antón and Gabriel Sala \\ Instituto de Energía Solar- UPM, Avenida Complutense, 28040, Madrid Spain \\ ${ }^{\text {a)} C o r r e s p o n d i n g ~ a u t h o r: ~ r u b e n . n u n e z @ i e s-d e f . u p m . e s ~}$
}

\begin{abstract}
Spectral variation of solar irradiance can be analyzed by the means of component cells, being useful for multi junction (MJ) and concentrator photovoltaics (CPV) characterization by the use of component cells derived spectral indexes, namely, spectral matching ratios (SMRs). When enough spectral data is available, a location can be also characterized and a MJ solar cell can be locally tuned. The AERONET network of ground-based sun photometers is employed to obtain the atmosphere parameters for 34 sites worldwide. To conclude, the SMR indexes are obtained for every location, and a comparative analysis is completed for four architectures of MJ solar cells technologies (two lattice match and two metamorphic). Based on this information, some cluster of locations that share similar spectral features arise.
\end{abstract}

\section{INTRODUCTION}

Concentrator photovoltaics (CPV) systems are based on multijunction (MJ) solar cells that are composed of several subcells connected in series. The benefit of MJ solar cells with respect single junction broadband cells is that each MJ subcell is optimized to a specific range of the solar spectrum and usually the MJ cell can absorb a broader spectral range. However due to the series connection, any change in solar spectrum with respect the reference spectrum (AM1.5D-G173-03) is going to provoke a current mismatch among the subcells and the MJ solar cell is only generating the minimum of the subcells currents. In fact, the most common scenario is that the photocurrent ratios $\left(\mathrm{I}_{\text {ratios }}\right)$ obtained at reference spectrum are only accomplished a very few times during a day or even not occurring. So the optimum $I_{\text {ratios }}$ that maximize every harvesting at a certain location may not be the ones that maximize the current of the device under AM1.5D-G173-03.

It can be useful to define a representative spectrum for a place, so this spectrum could be used to optimize a MJ solar cell and therefore a CPV module, for example, by tuning the photocurrents generated by every subcell [1] or by adjusting the optical design [2]. However this spectral information is scarce and is found only for a few places.

\section{Spectral Matching Ratio}

The Spectral Matching Ratio (SMR) quantifies a particular irradiance spectrum by comparing it to a reference spectrum, i.e. AM1.5D-G173-03. SMR is defined as the ratio between the currents photogenerated by two subcells under the spectrum in study, divided by the same ratio under the reference spectrum [3,4], and can be calculated as:

$$
S M R_{\text {subcell }_{j}}^{\text {subcell }_{i}}=\frac{I_{L, \text { ratio }_{\text {subcell }}}^{\text {subcel }_{i}}}{I_{L, \text { ratio }_{\text {subcell }_{j}} \text { ref }}}=\frac{\frac{I_{L, \text { subcell }_{i}}}{I_{L, \text { subcell }_{j}}}}{\frac{I_{L, \text { subcell }} \text { ref }_{\text {ref }}}{I_{L, \text { subcell }_{\text {ref }}}}}
$$


where $I_{L, \text { subcell }}$ represents the photocurrent of subcell i when illuminated with a particular spectral irradiance

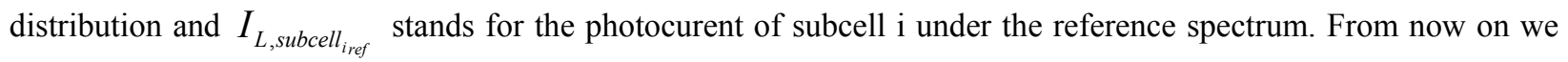
will assume that the aforementioned MJ solar cell and the component cells used to determine the set of photocurrents and consequently SMRs are of the same technology, i.e., they have the same relative spectral responses. The component cells provide a very low spectral resolution, typically composed of three values, but enough to determine the prevailing spectral conditions and to estimate the performance of state-of-the-art CPV technologies $[5,6]$.

A procedure for the spectral characterization of a particular site based on SMR indexes is proposed and then used to characterize spectrally worldwide locations. This information reveals how the annual energy weighted spectrum of the site compares to the reference spectrum and provides useful information for the optimization of a particular MJ technology for that site. To quantify the spectral losses due to spectral mismatch of a location with regard to reference spectrum, the derived spectral efficiency computes the losses associated to such spectral variations. Moreover, its integration over days, months or years for a specific site reveals the spectral losses to be expected in such site for a particular MJ technology, which can be used in the modeling and energy forecast of CPV systems.

\section{ANNUAL SPECTRAL ENERGY DISTRIBUTION VERSUS SMR}

Strongly influenced by local climate, the majority of the energy may be found at SMR values different from one, e.g., at high altitude locations with clear sky the most probable situation is $S M R_{\text {mid }}^{\text {top }}>1$. Therefore the middle subcell would limit the current of the device most of the time in the case that the bottom subcell supplies excess of current (as in the case of lattice-matched (LM) MJ solar cells based in germanium). As a consequence, the $I_{L, \text { ratio } m i d \text { ref }}^{\text {top }}$ value that maximizes the annually harvested energy for that particular location would be lower than one.

The SMRs representative for a place can be calculated by integrating the SMRs weighted by the direct normal irradiation $\mathrm{E}_{\mathrm{DNI}}$, over the whole year:

$$
\overline{S M R}_{\text {subcell }_{j}}^{\text {subcell }_{i}}=\frac{\int_{\text {year }} \operatorname{SMR}_{\text {subcell }_{j}}^{\text {subcell }_{i}}(t) \cdot E_{D N I}(t) d t}{\int_{\text {year }} E_{D N I}(t) d t}
$$

The distribution of the annual direct normal irradiation as a function of $\mathrm{SMR}_{\mathrm{mid}}^{\text {top }}$ and $\mathrm{SMR}_{\mathrm{bot}}^{\mathrm{mid}}$ is shown in Fig. 1 for Madrid in 2013 with minute resolution. Both SMRs define a mesh whose size is 100x100 bins with a resolution of $0.05 \times 0.05$. The color map represents an indication of the direct normal irradiation that is received in Madrid for every combination of SMR values.

The black dot in Fig. 1 indicates the SMRs barycenter, that is, the annually DNI-weighted in spectral coordinates $\left(\overline{S M R}_{\text {mid }}^{\text {top }}\right.$ and $\overline{S M R}_{\text {bot }}^{\text {mid }}$ ) which are respectively 0.96 and 1.00 . It must be pointed out that both values are very close or equal to one, which indicates that the reference spectrum AM1.5D-G173-03 is quite suitable to optimize a $\mathrm{LM} \mathrm{GaInP/GaInAs/Ge} \mathrm{MJ} \mathrm{solar} \mathrm{cell} \mathrm{for} \mathrm{the} \mathrm{climate} \mathrm{of} \mathrm{Madrid.}$ 


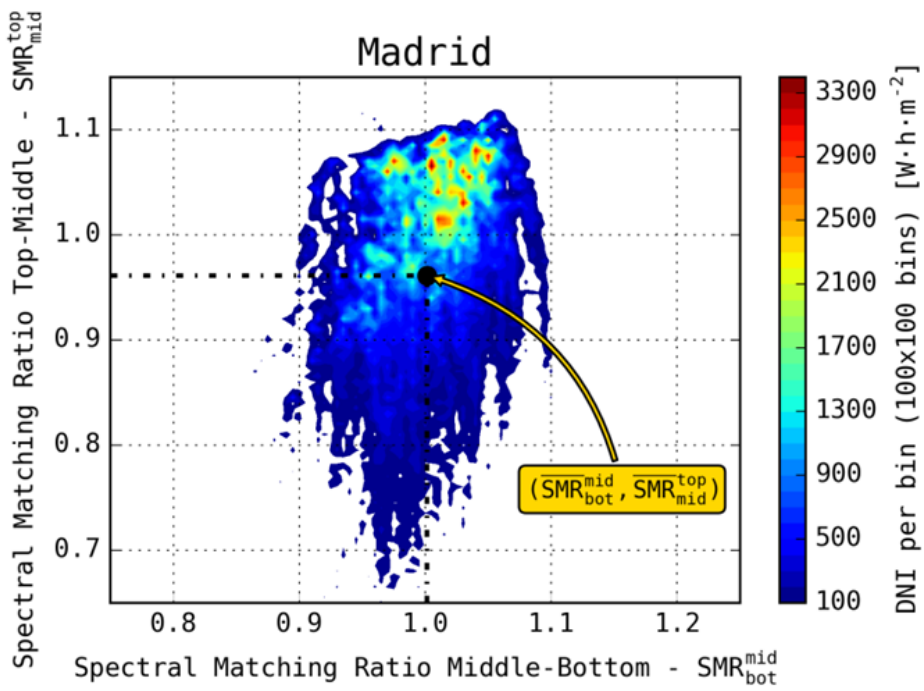

FIGURE 1. Spectral distribution of direct normal irradiation in Madrid throughout 2013 expressed as a function of spectral matching ratios ( $S M R_{\text {mid }}^{\text {top }}$ and $S M R_{\text {bot }}^{\text {mid }}$ ). The black dot indicates the barycenter, i.e. the DNI weighted SMR values that minimize the annually integrated spectral losses $\left(\overline{S M R}_{\text {mid }}^{\text {top }}\right.$ and $\left.\overline{S M R}_{b o t}^{\text {mid }}\right)$.

A graphical representation of the distribution of the annual DNI as a function of $S M R_{\text {mid }}^{\text {top }}$ and $S M R_{b o t}^{\text {mid }}$ has been proposed for the spectral characterization of a particular site for CPV applications [7], whose coordinates $\overline{S M R}_{\text {mid }}^{\text {top }}$ and $\overline{S M R}_{\text {bot }}^{\text {mid }}$ reveal how well matched is a particular MJ technology to that site and the current balance correction needed to optimize that technology to the site.

\section{SYNTHETIC WORLD SPECTRAL NETWORK}

This procedure can be extended to different worldwide climates. Since nowadays the amount of deployed spectroheliometers around the globe is quite reduced, another approach considering synthetic spectra can be an interesting option for the atmosphere characterization. We propose an approach that involves the use of atmospheric radiative transfer models, such as SMARTS, SPECTRAL2, and MODTRAN fed by spectral parameters. Among the spectral factors, the sunlight path length, which is function of Sun's zenith angle and altitude, the aerosol extinction and water vapor absorption are considered to be the principle impact factors [8].

Furthermore, a representative number of simulated spectral irradiance distributions can be used for estimating the average effect of the spectral variations in the performance of a MJ solar cell in periods of days, months, or a whole year for a specific site. This information can be used for the optimization of the current balance between subcells within a MJ solar cell under the standard reference spectrum, which will depend on the site.

AERONET is a network of ground-based sun photometers that contains spectral parameters information. The set of spectrally-resolved DNI for a number of sites and years, modeled with SMARTS from AERONET data, has been used to obtain SMR spectral indexes $[9,10]$ used in CPV considering a representative operation cell temperature of $90{ }^{\circ} \mathrm{C}$. However an analysis of the influence in a concrete CPV system should also consider the effect of the optics on the spectral distribution on the MJ cell [11], the change on the transmittance due to temperature variations on the optics [12] and ambient temperature fluctuations.

\section{Data Description}

The AERONET database contains data of more than 800 locations, whose measured atmospheric parameters have different level of quality for each location and every year. The site and period selection criteria are set to be:

- A particular year must have at least a certain number of data points, fixed at 10000; 
- Data must be available during each month of a year. All the sites are expected to have at least one sunny day per month even in winter conditions;

- $\quad$ Altitude of the site not exceeding $2000 \mathrm{~m}$;

- $\quad$ Latitude of site not exceeding 50 degrees.

To avoid a too limited set of sites, we have not considered an annual DNI threshold (even if some of them cannot be suitable for $\mathrm{CPV}$ ). Nevertheless all of them provide valuable information about spectral differences. Under these criteria, 34 locations with at least one year of data were selected, covering a wide range of climate conditions.

\section{Experimental Validation. Case Study: Madrid}

IES-UPM has been collecting spectroheliometer SMR data at a site that is only $200 \mathrm{~m}$ distant from an AERONET station maintained by the Spanish state meteorological agency (AEMET). Both instruments see virtually the same sky, which represents a convenient scenario for comparing both spectral data sources.

Considering that AERONET stations usually have a sampling frequency of 15 minutes and the spectroheliometer is sampled every minute, the spectroheliometer dataset has been interpolated to the time array of the AERONET data. Regarding data screening, AERONET level 2.0 data quality has been selected, therefore it assures cloud screening plus manual quality verification. The coordinates $\overline{S M R}_{\text {mid }}^{\text {top }}$ and $\overline{S M R}_{\text {bot }}^{\text {mid }}$ are respectively 0.96 and 1.00 obtained from the spectroheliometer measurements and 0.94 and 0.98 from the AERONET atmospheric data and simulated spectrally resolved DNI, which shows a reasonably good agreement between both procedures.

\section{Mapping the Solar Resource with the Spectral Characteristics of Worldwide Locations}

Among the atmospheric parameters, air mass (AM) corrected with site elevation [13], aerosol optical depth (AOD), and precipitable water (PW) are considered to be the main factors impacting DNI spectrum, while others such as ambient temperature, air pressure, ozone and others have a minor impact [14]. Nevertheless, the impact of such atmospheric condition on the MJ device performance cannot be directly calculated, but it is necessary to estimate the spectral distribution of DNI first by means of a radiative transfer model and then calculate the performance of the device through its spectral response. Alternatively, SMR indexes allow the direct estimation of the impact on the MJ performance since they account for the variations in current ratios among the subcells of the device. Thus, it would be helpful to determine relationships between the atmospheric indexes (AM, AOD, PW) and the spectral indexes (SMRs).

We shall start studying the atmospheric parameters that affects each annual DNI-weighted averages $\overline{S M R}_{\text {mid }}^{\text {top }}$ and $\overline{S M R}_{b o t}^{\text {mid }}$. For this case, we shall use the annual DNI-weighted averages of the atmospheric parameters as input values, i.e. $\overline{A M} \overline{A O D}$, and $\overline{P W}$, instead of their instant values. 


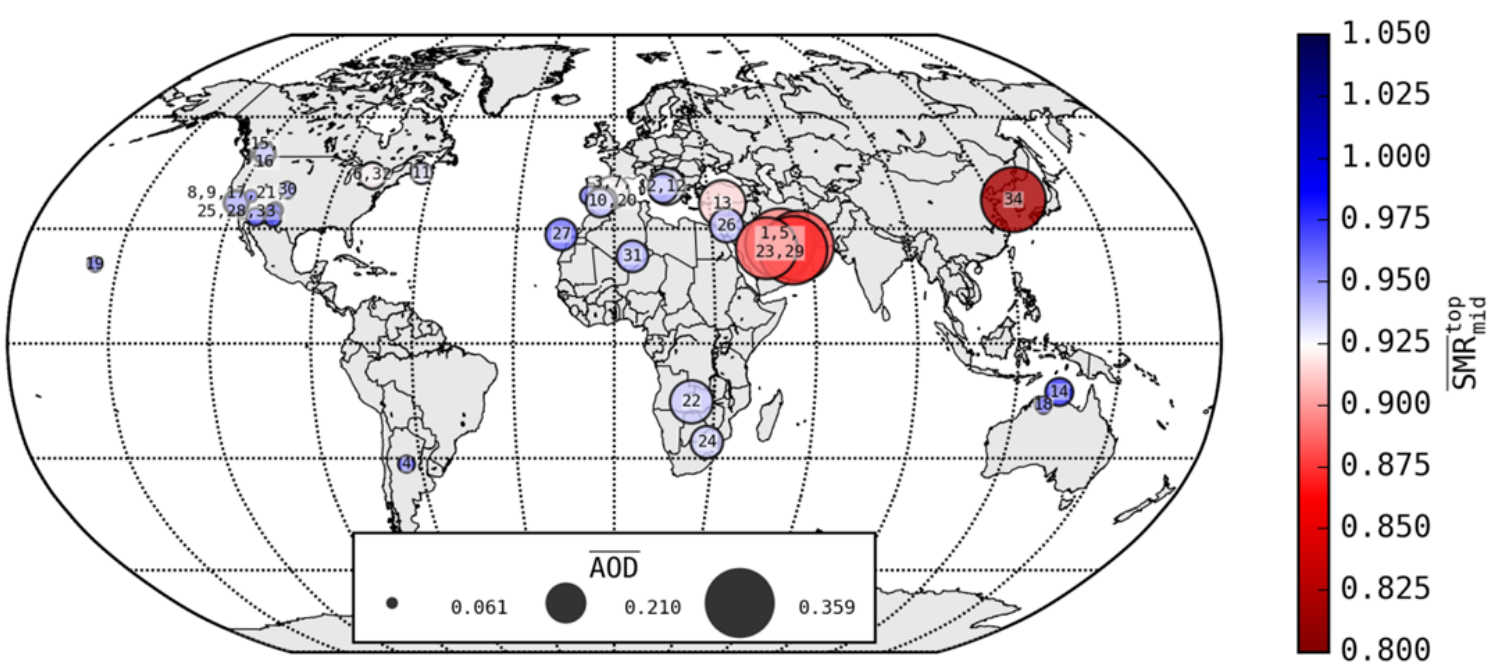

FIGURE 2. World distribution of selected AERONET locations showing both their annual DNI-weighted mean spectral matching ratio between top and middle subcells $\left(\overline{S M R}_{\text {mid }}^{\text {top }}\right)$ and their annual DNI-weighted mean aerosol optical depth $\overline{A O D}$. $\overline{S M R}_{\text {mid }}^{\text {top }}$ is represented in a color scale while a diameter scale is used for $\overline{A O D}$. Note: numbers inside circles identify AERONET locations and can be found in the list of Appendix.

The $S M R_{\text {mid }}^{\text {top }}$ index covers the top and middle subcell spectral regions, i.e., from 350 to $900 \mathrm{~nm}$, so it is mainly affected by AM and AOD [10]. The lower the latitude, the lower the mean air mass, which result in higher values of $\overline{S M R}_{\text {mid }}^{\text {top }}$. The 34 values of $\overline{S M R}_{\text {mid }}^{\text {top }}$ for the studied sites are represented in a global map (Fig. 2) as circles with a color scale together with the corresponding $\overline{A O D}$ through the circle diameter, showing a clear relationship between both parameters. In general, low $\overline{A O D}$ sites (small dots) show higher $\overline{S M R}_{\text {mid }}^{\text {top }}$ values (blue dots) as Lanai in Hawaii (19), while high $\overline{A O D}$ sites (large dots) show low $\overline{S M R}_{\text {mid }}^{\text {top }}$ values (red dots) as the Arabian Desert (1, 5, 23 and 29). 


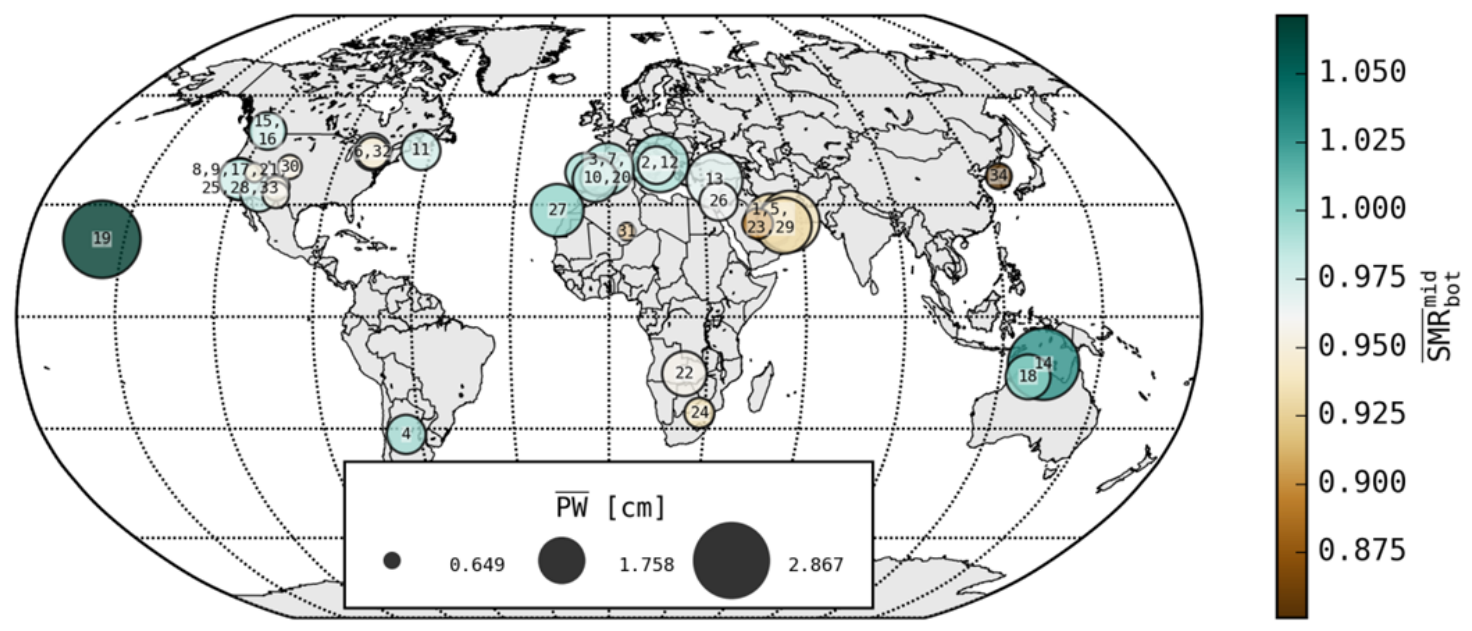

FIGURE 3. World distribution of selected AERONET locations showing both their annually DNI-weighted Spectral Matching Ratio between Middle and Bottom subcells ( $\left.\overline{S M R}_{b o t}^{\text {mid }}\right)$ and their annually DNI-weighted Precipitable Water $(\overline{P W}) . \overline{S M R}$ mot is represented in a color scale while a diameter scale is used for $\overline{P W}$. Note: numbers inside circles identify AERONET locations and can be found in the list of Appendix.

The relationship of $\overline{S M R}_{b o t}^{\text {mid }}$ with $\overline{\mathrm{PW}}$ is pointed out in Fig. 3. The higher the $\overline{\mathrm{PW}}$, the stronger the absorption of the DNI in the infrared region, more important in the bottom subcell region than in the middle one, leading to high $S M R_{b o t}^{\text {mid }}$ values. Consequently, the proximity of big masses of water (as in the case of islands and coastal sites) increases the level of $\overline{S M R}_{b o t}^{\text {mid }}$; some example cases are Lanai (19) in Hawaii and Jabiru (14) and Lake Argyle (18) in Australia. On the other hand, especially dry sites, such as the North American South West $(25,28,30$ and 33) or in inner deserts show very low $\overline{S M R}_{b o t}^{\text {mid }}$, with Tamanrasset (31) being a good example.

\section{GLOBAL ANALYSIS OF SPECTRAL INFLUENCE FOR SEVERAL KINDS OF TRIPLE JUNCTION CELLS}

The previous work was supposing a LM GaInP/GaInAs/Ge triple junction solar cells, hereafter LM-Ge. The aim of this part is to compare these data with those obtained for other triple junction technologies, including various types of lattice-matched and metamorphic triple-junction solar cells, covering on the one hand lattice-matched $3 \mathrm{~J}$ solar cells, both traditional cells based on germanium substrates and cells based on dilute nitrides, and on the other hand metamorphic $3 \mathrm{~J}$ solar cells, both upright and inverted grown.

Therefore same calculation detailed above has been carried out for the other three types of solar cells (UMM, IMM and LM-DN). Figure 4 shows $\overline{S M R}_{\text {mid }}^{\text {top }}$ and $\overline{S M R}_{b o t}^{\text {mid }}$ for the four cell technologies. The differences between the cell technologies is observed to be relatively low compared to the strong differences between locations, which is a consequence of the high degree of similarity between the spectral range of top, middle and bottom subcells for the four technologies. Some sites have geographic parameters in common and form clusters that have similar levels of annual DNI-weighted spectral parameters relevant to highlight as those in deserts, close to big masses of water and North-American South-West.

Regarding $\overline{S M R}_{\text {mid }}^{\text {top }}$ the differences among the technologies are below $1 \%$ for half of the sites and between $1 \%$ and $2 \%$ for the other half, while the differences among sites range about $20 \%$, from 0.82 to 1.03 . 


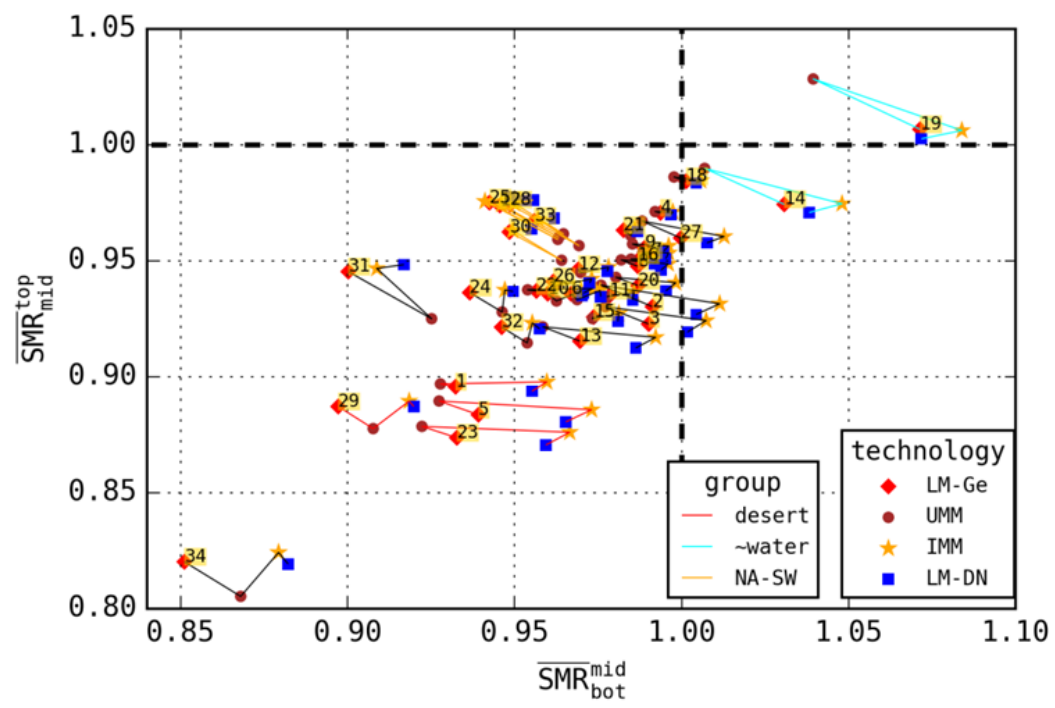

FIGURE 4. Annually DNI-weighted Spectral Matching Ratio between top and middle subcells ( $\left.\overline{S M R}_{\text {mid }}^{t o p}\right)$ and between middle and bottom subcells ( $\overline{S M R}$ bot $)$ for the selected locations considering four different MJ technologies. Some sites have geographic parameters in common and form groups that have similar levels of annual DNI-weighted spectral parameters relevant to highlight as those in deserts (desert), close to big masses of water ( water) and North-American South-West (NA-SW). Note: numbers inside circles identify AERONET locations and can be found in the list of Appendix.

In a similar way, Fig. 4 also shows $\overline{S M R}_{b o t}^{\text {mid }}$ for the 34 sites in the horizontal axis. In this case the differences among the 4 cell technologies are higher which correspond with the greater differences in the spectral responses of the 4 bottom subcells. About $60 \%$ of the sites show differences below $2 \%$, while the others have values up to $4.5 \%$. Nevertheless the differences among sites are again much higher and vary more than $20 \%$.

A majority of sites show also a $\overline{S M R}_{\text {bot }}^{\text {mid }}$ value below one but in this case there are many sites with values close to one or above. Nevertheless, the impact of the $\overline{S M R}_{b o t}^{\text {mid }}$ depends very much on the cell technology, since the current of the bottom subcells exceeds the other two from only about $7 \%$ for the case of the LM-DN cells to more than $40 \%$ for the LM-Ge subcells.

It is worth making the point that the distance among SMR coordinates of different technologies of a given site is related to the closeness of its atmosphere to AM1.5D-G173 conditions. The closer is a location to the ideal situation $\left(\overline{S M R}_{\text {mid }}^{\text {top }}=1\right.$ and $\overline{S M R}_{\text {bot }}^{\text {mid }}=1$ ), the closer are the four SMR coordinates. Sites with high/low $\overline{A O D} / \overline{P W}$ as Yonsey_University (34) $(\overline{A O D}=0.33, \overline{P W}=0.96)$ and low/high $\overline{A O D}$ as Lanai (19) $(\overline{A O D}=0.08, \overline{P W}$ $=2.87$ ) highlight those differences which are caused by the combination of the cut-off wavelengths of the subcells and the dominant levels of atmospheric components of a site.

\section{CONCLUSIONS}

Several capabilities of the SMR as spectral index for CPV applications have been shown in this paper beyond their most common use in the CPV community, which is filtering of operating conditions to fix a certain spectral condition. First, SMR indexes have been used to characterize the solar resource at a specific site through their annual DNI-weighted average values related to a MJ solar cell technology. This provides the spectral information that gives most of the necessary information for optimizing the spectral response of a CPV module (whose response at a reference site is known) for a new location. This may be carried out by tuning the current ratio of the receiver multi-junction cells by a factor corresponding to the difference in SMR between the known site and the new site or considering external factors as optics. 
Then, the relationships between SMR indexes and spectral parameters, mainly AM, AOD, and PW have been determined for annual DNI-weighted averages, which allows the prediction of the SMR indexes if some geographic and atmosphere parameters of a site are known. The capability of the AERONET network to provide such needed parameters has been exploited in this study, selecting 34 worldwide sites with the best data quality and covering many different climate conditions.

Finally, the site characterization by means of SMR indexes have been extended to several triple-junction technologies, covering lattice-matched cells based on germanium and dilute nitrides and both inverted and upright metamorphic cells. The comparative analysis shows large differences in the performance of the devices between the selected locations, while the differences among the technologies were much less significant. When all the sites are plotted in the SMR diagram most of them are placed in the $\overline{S M R}_{\text {mid }}^{\text {top }} \& \overline{S M R}_{\text {bot }}^{\text {mid }}<1$ quadrant, suggesting that the reference spectrum is not representative and MJ solar cells should be adapted to local circumstances.

\section{APPENDIX}

List of analyzed AERONET stations: 1-Bahrain, 2-Bari_University, 3-Burjassot, 4-Cordoba-CETT, 5-Dhadnah, 6-Egbert, 7-Evora, 8-Fresno, 9-Fresno_2, 10-Granada, 11-Halifax, 12-IMAA_Potenza, 13-IMS-METU-ERDEMLI, 14-Jabiru, 15-Kelowna, 16-Kelowna_UAS, 17-Kirtland_AFB, 18-Lake_Argyle, 19-Lanai, 20-Malaga, 21-Maricopa, 22-Mongu, 23-Mussafa, 24-Pretoria_CSIR-DPSS, 25-Railroad_Valley, 26-Santa_Cruz_Tenerife, 27SEDE_BOKER, 28-Sevilleta, 29-Solar_Village, 30-Table_Mountain, 31-Tamanrasset_INM, 32-Toronto, 33White_Sands_HELSTF, 34-Yonsei_University.

\section{ACKNOWLEDGMENTS}

The authors want to thank the AERONET network maintainers. This work has been partially supported by This work has been supported by the Spanish Ministerio de Economia y Competitividad (MINECO) under the program RETOS-COLABORACIÓN (RTC-2015-3982-3) and I+D-Excelencia 2013 ENE2013-45299-P. This work made use of the Scipy stack [15], an open-source Python-based scientific computing environment.

\section{REFERENCES}

1. $\quad$ K. Araki and M. Yamaguchi, Sol. Energy Mater. Sol. Cells 75, 707 (2003).

2. M. Victoria, S. Askins, R. Núñez, C. Domínguez, R. Herrero, I. Antón, G. Sala, and J.M. Ruíz, in AIP Conf. Proc. (2013), p. 156.

3. C. Domínguez, I. Antón, G. Sala, and S. Askins, Prog. Photovolt. Res. Appl. 21, 1478 (2013).

4. R. Núñez, C. Domínguez, S. Askins, M. Victoria, R. Herrero, I. Antón, and G. Sala, Prog. Photovolt. Res. Appl. 24, 663 (2016).

5. G. Siefer, M. Steiner, M. Baudrit, C. Dominguez, I. Antón, R. Núñez, F. Roca, P.M. Pugliatti, A.D. Stefano, R. Kenny, and P. Morabito, in AIP Conf. Proc. (AIP Publishing, 2014), pp. 167-172.

6. I. Antón, M. Martínez, F. Rubio, R. Núñez, R. Herrero, C. Domínguez, M. Victoria, S. Askins, and G. Sala, in AIP Conf. Proc. (2012), pp. 331-335.

7. R. Núñez, I. Antón, S. Askins, G. Sala, C. Domínguez, P. Voarino, M. Steiner, G. Siefer, R. Fucci, F. Roca, A. Minuto, and P. Morabito, in AIP Conf. Proc. (AIP Publishing, 2015), p. 030006.

8. C.A. Gueymard, in Opt. Model. Meas. Sol. Energy Syst. III (2009), pp. 741007-741007-14.

9. J. Jaus and C.A. Gueymard, in AIP Conf. Proc. (2012), pp. 122-126.

10. R. Núñez, I. Antón, S. Askins, and G. Sala, in AIP Conf. Proc. (AIP Publishing, 2014), pp. 290-293.

11. M. Victoria, R. Herrero, C. Domínguez, S. Askins, I. Antón, and G. Sala, in 25th Eur. Photovolt. Sol. Energy Conf. Exhib. - 5th World Conf. Photovolt. Energy Convers., edited by IE-JRC, DG-JRC, and WIP (WIP Munich, Valencia, Spain, 2010), pp. 143-146.

12. S. Askins, M. Victoria, R. Herrero, C. Domínguez, I. Antón, G. Sala, F. Dimroth, S. Kurtz, G. Sala, and A.W. Bett, in AIP Conf. Proc. (2011), pp. 57-60.

13. F. Kasten and A.T. Young, Appl. Opt. 28, 4735 (1989).

14. M. Muller, B. Marion, S. Kurtz, and J. Rodriguez, in AIP Conf. Proc. (2010), p. 307.

15. F. Pérez, B.E. Granger, and J.D. Hunter, Comput. Sci. Eng. 13, 13 (2011). 